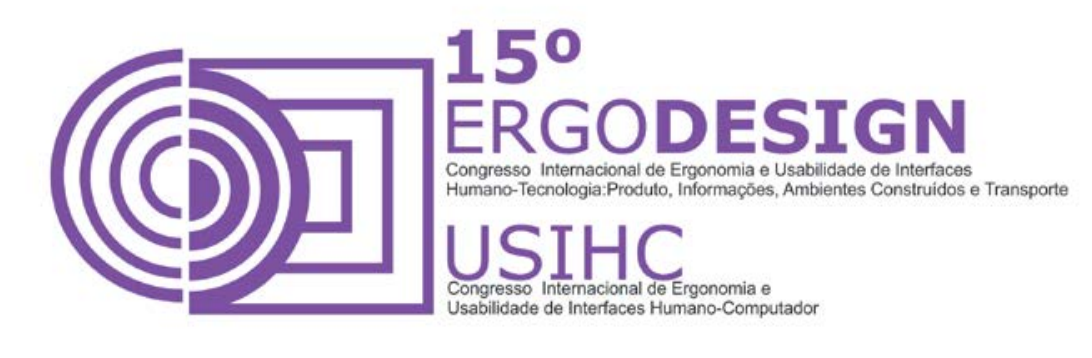

\title{
INTERFACE PARA SISTEMA DE COMUNICAÇÃO ONLINE PARA A UNIVERSIDADE FEDERAL DO AMAZONAS
}

\section{INTERFACE FOR ONLINE COMMUNICATION SYSTEM FOR UNIVERSIDADE FEDERAL DO AMAZONAS}

\author{
CARDOSO, Vânia Maria Batalha Cardoso (1) \\ ARAÚJO, Leandro César Melo (2) \\ VERGARA, Lizandra G. Lupi (3) \\ (1) Universidade Federal de Santa Catarina, Mestre \\ e-mail: vania.batalha60@gmail.com \\ (2) Universidade Federal do Amazonas, Graduado \\ e-mail: leandro.cesar.ma@gmail.com \\ (3) Universidade Federal de Santa Catarina, Doutora \\ e-mail: lizandravergara@gmail.com
}

\begin{abstract}
RESUMO
Esta pesquisa propõe a criação de uma ferramenta de comunicação online entre acadêmicos e professores de uma Universidade Federal. A internet facilita e agiliza a comunicação e através dela também é possível organizar atividades acadêmicas como compartilhamento de arquivos. Entretanto, as ferramentas virtuais disponibilizadas pela instituição não permitem a comunicação extraclasse entre acadêmicos e professores. Através de pesquisa bibliográfica, problematização e análise do sistema atual, inquirição com usuários e análise de similares, requisitos e parâmetros, chega-se a uma interface em forma de rede social, sistema online, que integra as principais funções existentes no sistema atual além de permitir a conexão extraclasse.

Palavras-chave:Usabilidade, Conexão Extraclasse, Acadêmicos, Professores.
\end{abstract}

\section{ABSTRACT}

This research proposes the creation of an online communication tool between students and teachers from a Federal University. The internet facilitates and streamlines communication and through it is also possible to organize academic activities such as file sharing. However, virtual tools provided by the institution do not allow extracurricular communication between academics and teachers. Through 


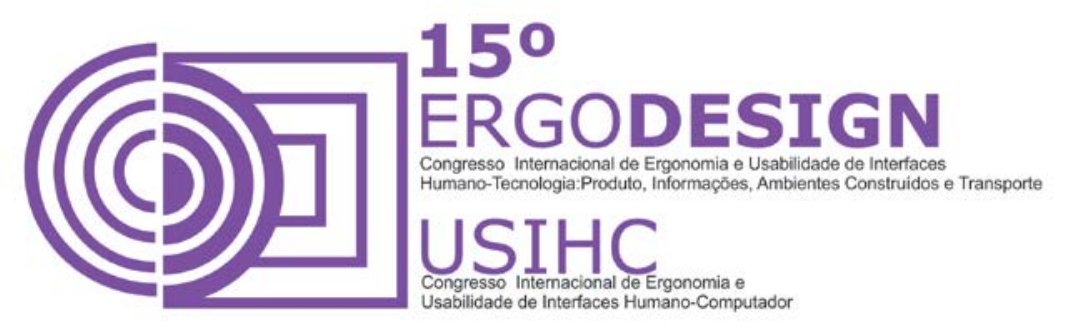

literature, questioning and analysis of the current system, users with hearing, similar analysis, requirements and parameters, we arrive at an interface in the form of social networking, online system that integrates key functions in the current system as well as allow extracurricular connection.

Keywords: Usability, Extracurricular Connection, Academics, Professors.

\section{INTRODUÇÃO}

A World Wide Web (Teia Mundial de Computadores) abre horizontes para cerca de 78 milhões (Ibope/Nielsen, 2011) de brasileiros todos os dias, onde seu principal foco é a comunicação. Desde 1997, o ano que deu início a grande expansão da internet, as pessoas se comunicam regularmente na internet. E com o surgimento das redes sociais virtuais, a comunicação passou por uma grande mudança.

Em 2010, em uma pesquisa feita pela F/Nazca Saatchi \& Saatchi (agência de publicidade brasileira), descobriu-se que $68 \%$ dos entrevistados passam a maior parte do seu tempo de navegação no Orkut, a rede social com mais usuários no Brasil.

$\mathrm{Na}$ Universidade Federal do Amazonas apenas são oferecidas aos professores e alunos ferramentas básicas e defasadas de matrícula e saldo de notas, quando se é possível oferecer juntamente a estas ferramentas funcionalidades como: controle geral do que se é estudado em sala de aula, calendário de atividades, ementas de matérias e tantas outras. Tais ferramentas geram grandes reclamações por parte de seus usuários. Em certos momentos o sistema fica indisponível e há casos de alunos que perderam seu cadastro na instituição, o que ocasiona um constrangimento para a UFAM e seus funcionários.

Portanto, este projeto pretende minimizar tais problemas oferecendo uma ferramenta de baixo custo de manutenção e de fácil manuseio, tendo por objetivo principal promover a comunicação online entre professores e acadêmicos da UFAM através do desenvolvimento de uma interface de rede social institucional, visando:

a) Viabilizar a Integração entre Portal do Aluno e Portal do Professor;

b) Agilizar a troca de arquivos, tais como exercícios, trabalhos, textos de ajuda e outros documentos de aula;

c) Oferecer suporte para as atividades entre acadêmicos e professores.

O desenvolvimento deste projeto conta com uma a pesquisa descritiva, onde foi utilizado o método cíclico proposto por BONFIM (1995) que tem como característica principal a possibilidade de retorno entre as etapas sem que as mesmas sejam predeterminadas ou obrigatórias.

A partir de pesquisa bibliográfica e de observações referentes ao funcionamento do sistema de comunicação digital atual - coleta de dados - faz-se a delimitação dos problemas, entrevistas, são levantados similares que serão analisados de acordo com o sistema alvo apresentado segundo modelagem sistêmica de MORAES \& MONT'ALVÃO (2010) e, posteriormente, realizase a análise dos dados. 


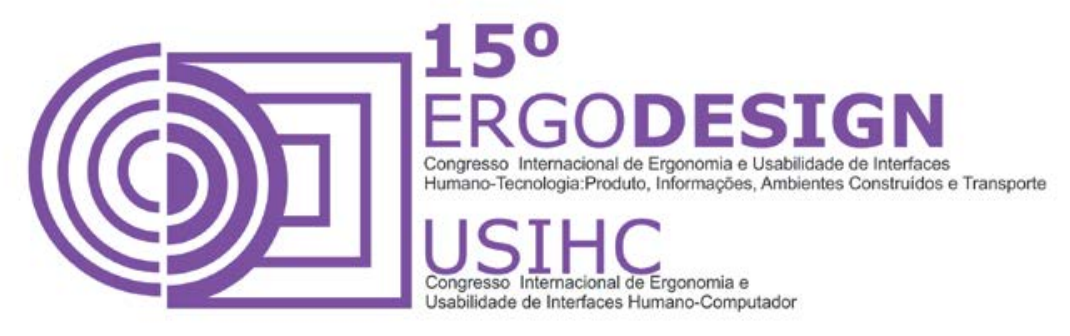

A partir dos problemas encontrados nos similares e o objetivo a ser atingido serão definidos os requisitos e os parâmetros para a criação das alternativas. Com as alternativas prontas foi realizada uma avaliação para que se possa definir qual alternativa cumpre todos os requisitos e o objetivo a atingir. Então se detalhará a alternativa.

\section{REFERENCIAL TEÓRICO}

\section{$2.1 \quad$ A internet}

A Internet é a união de milhões de conexões de rede que permite o acesso a informações e a transferência de dados por meio do TCP/IP (infra). Ela oferece uma ampla gama de funções e usos, tal qual correio eletrônico, bate-papos, troca de arquivos, grupos de notícias ou fóruns, a WWW e vários outros. Por causa de seus recursos passou a ser o meio de comunicação mais usado atualmente. Dentro dela é possível armazenar as informações pessoais de quem a está usando, quase que totalmente. Conhecer suas funções, recursos e funcionamento, é imprescindível para o desenvolvimento de novas ferramentas, pois assim, se conhecerá todas as possibilidades oferecidas por ela.

A internet funciona por meio de três camadas, chamadas protocolos. Na camada inferior está o Protocolo de Internet (em inglês, Internet Protocol), ou IP. O IP é quem define os pacotes que carregam blocos de dados de um nó de rede para outros que estão interconectados na camada média. Na camada média estão os protocolos que controlam a transmissão de dados, são eles o Transmission Control Protocol (TCP), o User Datagram Protocol (UDP) e o Internet Control Message Protocol (ICMP). E na camada mais acima estão os protocolos na qual o usuário tem mais contato. Nele se encontra o DNS (Domain Name Service ou Serviço de Nome de Domínios), estre é responsável pela tradução do endereço IP para um nome de domínio e viceversa. Também se encontra o HTTP (Hypertext Transfer Protocol, ou Protocolo de Transferências de Hipertexto), um protocolo de comunicação, coordenado pela World Wide Web Consortium (W3C) e pela Internet Engineering Task Force (IETF, Força Tarefa de Engenharia de Internet, em tradução livre), que é usado para a comunicação com sítios web (sites), quais usam a linguagem de marcação HTML (HyperText Markup Language, Linguagem de Marcação de HTML), porém a comunicação é feita com outros comandos distintos do HTML.

\subsection{Arquitetura da informação}

Para Wurman (1991), a informação deve ser aquilo que leva a compreensão. Atualmente o homem recebe demasiada informação que não consegue compreender todas. O Instituto de Arquitetura de Informação define Arquitetura de Informação como a arte e a ciência de organizar e catalogar websites, intranets, comunidades online e software de modo que a usabilidade seja garantida.

Van Dijck (2003) diz que a Arquitetura de Informação é um novo campo no webdesign onde focaliza a estrutura do website e não a funcionalidade ou o aspecto, o que a torna diferente do 


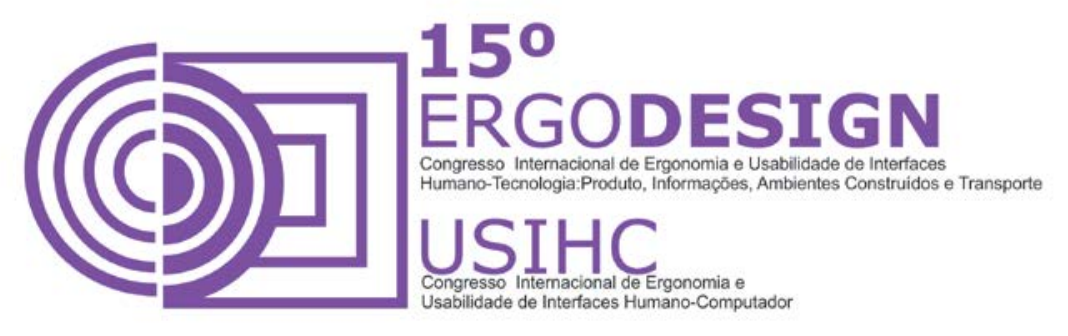

design e da programação visual. E que seu objetivo é criar websites de fácil uso, que preencham as necessidades e objetivos dos usuários.

A arquitetura de informação é a categorização da informação estruturada de forma coerente, onde a maioria dos receptores a possa entender. Pode-se exemplificar o trabalho do arquiteto de informação como sendo o de um bibliotecário, onde o bibliotecário arrumará os livros de uma biblioteca por autor, assunto ou de outra forma que seja da maneira mais fácil de encontrá-lo. Por isso, segundo Rosenfeld e Morville (1998), o arquiteto da informação deve ter conhecimentos que englobem o design gráfico, a ciência da informação e biblioteconomia, 0 jornalismo, a engenharia da usabilidade, o marketing e a ciência da computação.

\subsection{Design de interação}

No mundo moderno, uma pessoa interage com diversos produtos a fim de facilitar o caminho até o seu objetivo. Mas, interagindo com esses produtos, essa pessoa pode perceber que nem todos são facilmente utilizáveis. Segundo Preece et al (2002) o objetivo do design de interação é o desenvolvimento de de produtos interativos, que são fáceis, funcionais, agradáveis de serem usados - segundo a perspectiva do usuário.

Pode-se dizer que o design de interação é o desenvolvimento de produtos que ajudem as pessoas no seu dia a dia e no seu espaço de trabalho, melhorando o trabalho, a comunicação e a interação. Winograd, 1997, apud Preece et al, 2002 descreve como "o desenvolvimento de espaços para a comunicação e interação humana".

Num processo de desenvolvimento de interação o designer precisa saber no que e onde será usado o produto. Além disso, realiza testes que mostrem o que o usuário faz enquanto interage com o produto. Estes testes são necessários, pois de acordo com Preece et al (2002), a adequação dos diferentes tipos de interfaces e arranjos dos dispositivos de entrada e saída dependes em quais tipos de atividades necessitam ser suportadas.

\section{$2.4 \quad$ Interface}

Há vários conceitos para interface, o dicionário traduz como um ponto no qual um sistema de computação termina e outro começa e traduz ainda como equipamento e programa projetados para tornar mais fácil e eficiente a comunicação dos usuários com as máquinas.

Uma interface de usuário é o conjunto das características de uma ferramenta complexa com as quais o usuário interage com a ferramenta. As interfaces possuem dois métodos: de entrada (ou input) a qual o usuário manipula o sistema, e o de saída (ou output) onde o sistema produz o efeito (resposta) à ação do usuário.

A interface é a parte mais importante num website. É através dela que o usuário receberá e controlará a informação. Portanto, de acordo com Cybis (2007) dificilmente uma mesma interface significará a mesma coisa para dois usuários distintos, já que interfaces constituem, fundamentalmente, sistemas abertos dos quais usuários são agentes ativos. Assim pode-se afirmar que a experiência da Interação Humano-Computador é individual e única (idem). 


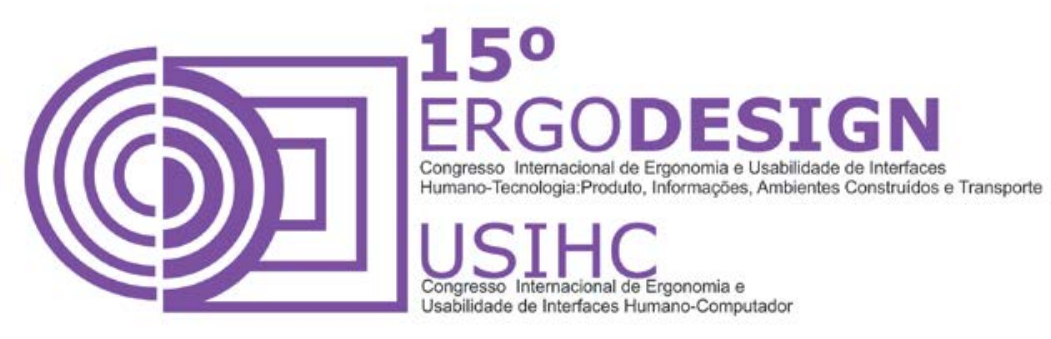

\subsection{Interação Humano Computador (IHC)}

Interação Humano-Computador, também conhecido como $\mathrm{IHC}$ ou $\mathrm{HCl}$ (Human-Computer Interaction), é o estudo da interação entre o homem e o computador, de forma multidisciplinar, onde essa interação é feita através da interface de usuário, o conjunto de software e hardware. A interface de usuário está presente desde a manipulação de um celular até a manipulação de grandes máquinas, por exemplo, porta aviões.

Segundo Moraes e Santa Rosa, o objetivo principal da área de interação humano-computador é o projeto e o desenvolvimento de sistemas com o propósito de melhorar a eficácia e proporcionar satisfação ao usuário. $\mathrm{O} \mathrm{HCl}$ tem como objetivo o aprimoramento de sistemas para que forneçam usabilidade ao usuário, isto é, fornecer ao usuário segurança, eficiência e segurança durante a execução de suas tarefas. Usabilidade é um termo relativamente novo, surgido entre a década de 80 e 90, que está ligada ao diálogo entre a interface do produto e o usuário. E para um produto ser considerado com boa usabilidade é necessário que ele tenha alguns aspectos como facilidade de aprendizagem, utilização eficiente, apresentar poucos erros e não acarretar em constrangimentos, como também pode estar ligada a facilidade de memorização e satisfação do usuário que também define a capacidade de se usar um sistema com facilidade para que a tarefa seja realizada com eficácia.

[...] a usabilidade é um atributo de todo produto - como a funcionalidade. Testar a funcionalidade significa certificar-se que o produto funciona de acordo com as especificações. [...] Testar a usabilidade significa ter certeza de que as pessoas podem reconhecer e interagir com funções que satisfaçam suas necessidades. (DUMAS \& REDISH, 1999 apud MORAES \& SANTA ROSA, 2008, p.14)

\section{PROBLEMATIZAÇÃO}

A UFAM apresenta dois sistemas de gerenciamento para professores e acadêmicos, são eles, respectivamente, o Portal do Professor e o Portal do Aluno. Estes dois sistemas são interligados e atuam diferentemente um do outro. Abaixo (Figura 1) é apresentado o organograma destes sistemas na UFAM:

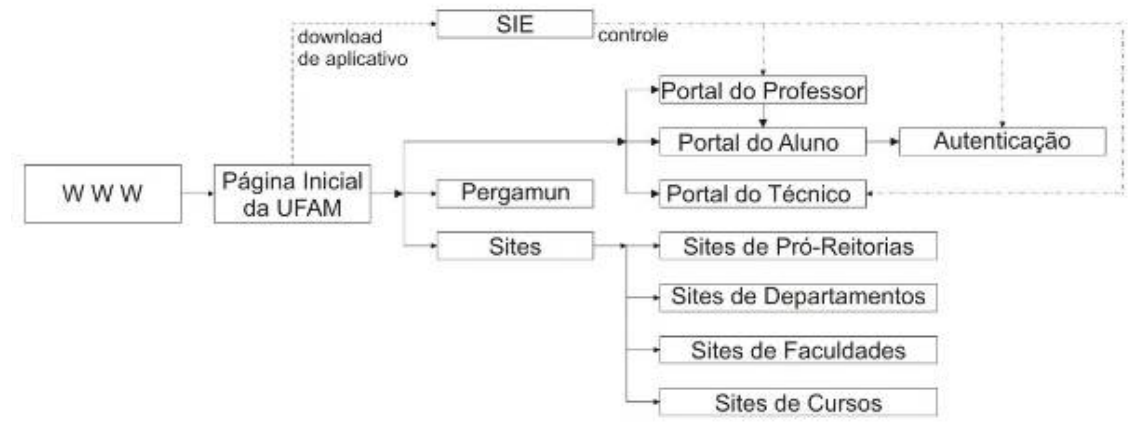




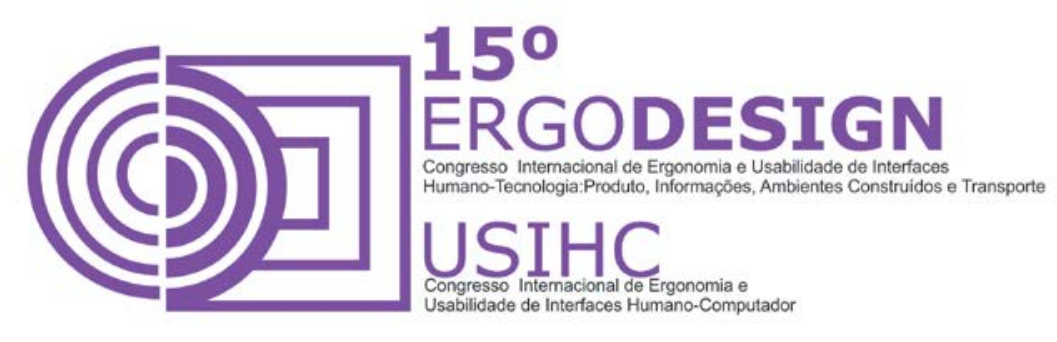

Figura 1 - Organograma do Portal do Professor e do Portal do Aluno

O Portal do Professor é ligado diretamente ao Portal do Aluno, enquanto o Portal do Aluno não possui ligação direta com o Portal do Professor. Essa ligação é oferecida apenas para o lançamento de notas de trabalhos e provas, onde o professor adiciona as notas no seu portal e o acadêmico as visualiza em seu portal.

No Portal do Professor são oferecidas as opções de lançamento de notas, visualização de horário, possibilidade de construir seu plano individual de trabalho e a visualização das avaliações feitas pelos seus alunos nos seus respectivos portais.

A interface do Portal do Professor é bem simples e limitada. Sem atrativo visual e com poucas funcionalidades, o professor não é convidado a retornar frequentemente ao portal.
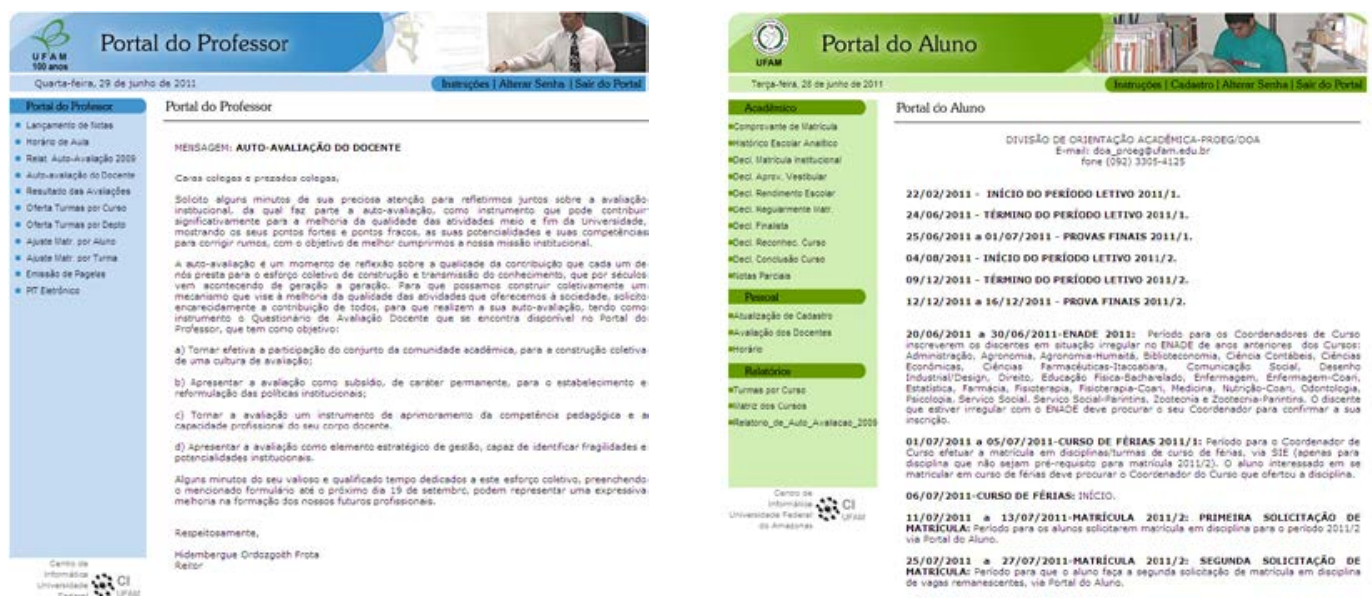

Figura 2 - Página Inicial do Portal do Professor e Portal do Aluno

\subsection{Caracterização e posição serial do sistema}

Segundo Meister (1976) apud Moraes e Mont'alvão (2010) os subsistemas podem ser arranjados em termos de uma posição serial de diferentes níveis, onde um subsistema pode ser completa ou parcialmente dependente, como pode ser independente, de entradas de outro subsistema para sua operação. O objetivo da caracterização e da posição serial de um sistema é saber como as informações são recebidas e enviadas, de que forma elas são processadas 


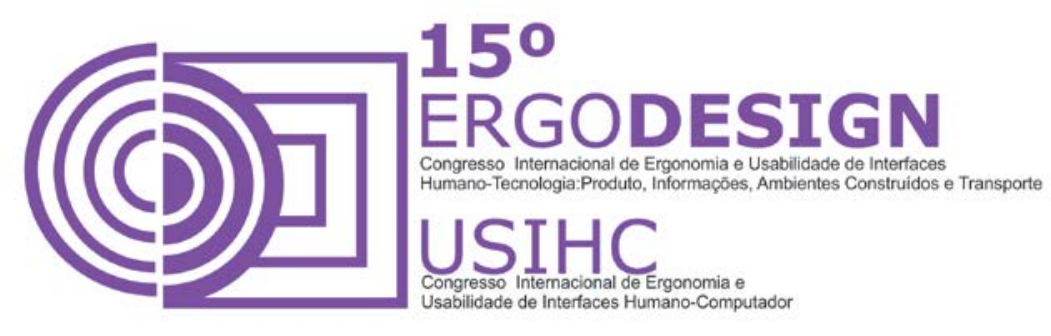

pelo sistema. Nas figuras a seguir serão apresentadas a caracterização do sistema (Figura 3) e a posição serial do sistema (Figura 4) segundo Moraes e Mont'alvão (2010).

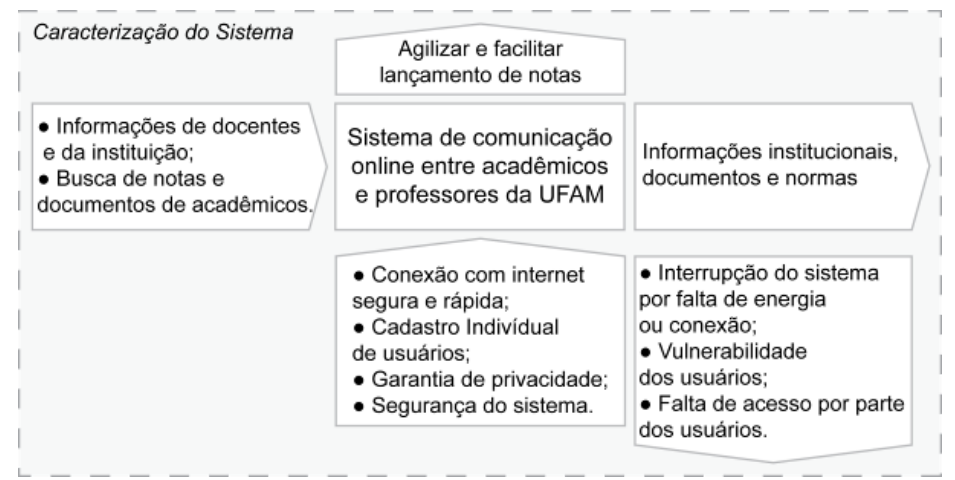

Figura 3 - Caracterização do Sistema

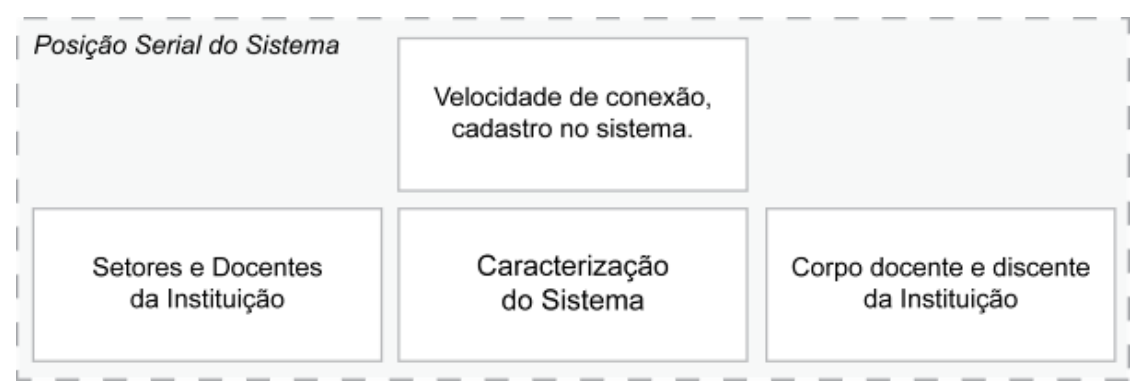

Figura 4 - Posição Serial do Sistema

O Portal do Aluno e o Portal do Professor foram implantados quando a UFAM começou a usar a internet para facilitar o trabalho internet. Estes portais agilizam as matrículas dos alunos, 0 lançamento de notas e retirada de documentos. Estas funções são pouco acessadas, o que ocorre principalmente no início e fim dos períodos de aulas.

Além de funções pouco acessadas o sistema não oferece atrativo visual, não oferecer funções mais relevantes para os usuários e ainda há usuários que perderam seu cadastro no Portal. Os portais sempre geraram reclamações, como relatos de lentidão no sistema - o que pode acontecer devido a grande quantidade de acessos simultâneos -, problemas de usabilidade informações difíceis de encontrar -, e erros no sistema - documentos que não se autenticam, erros de servidor e outros.

\subsection{Inquirições}

Foram feitas inquirições com acadêmicos e professores da UFAM a fim de conhecer quais ferra-mentas são mais utilizadas dentro e fora da instituição. 59 inquirições foram respondidas 


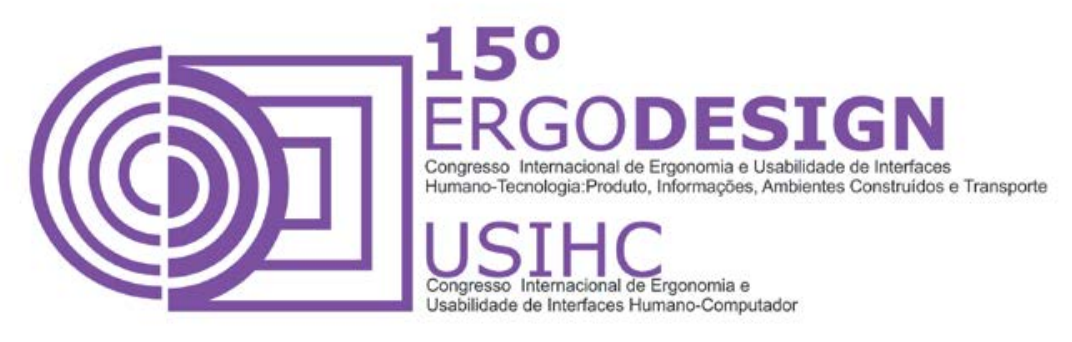

via inter-net com apoio da ferramenta "Google Docs Spreadsheet", sendo 40 acadêmicos e 19 professores. Foram feitas perguntas sobre se os indagados sabem usar um computador e a internet, para que eles usam o computador e a internet, como a internet é usada e quais ferramentas são usadas.

É possível perceber pelas respostas ao questionário, acadêmicos e professores buscam ferramentas externas a instituição para suprir a necessidade da comunicação extraclasse com seus professores. As ferramentas internas não oferecem recursos que facilitariam não só a

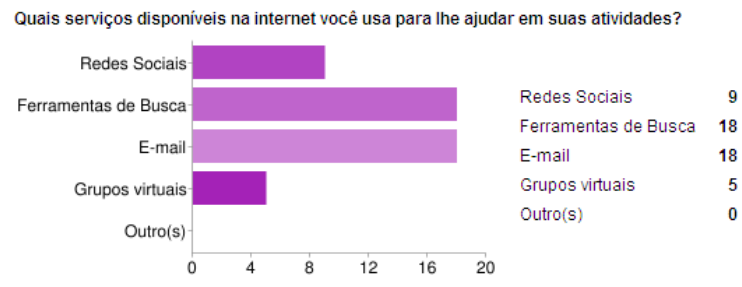

em sala de aula, trabalhos e provas. Oferecer essas funções é um dos objetivos desse trabalho. comunicação como também o ensino em si, permitindo maior controle do que é apresentado

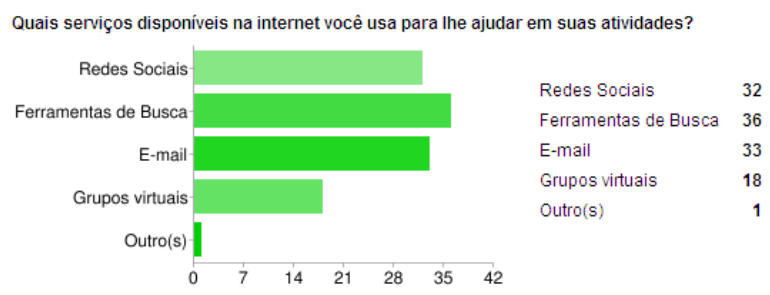

Figura 5 - Utilização de serviços por parte dos professores
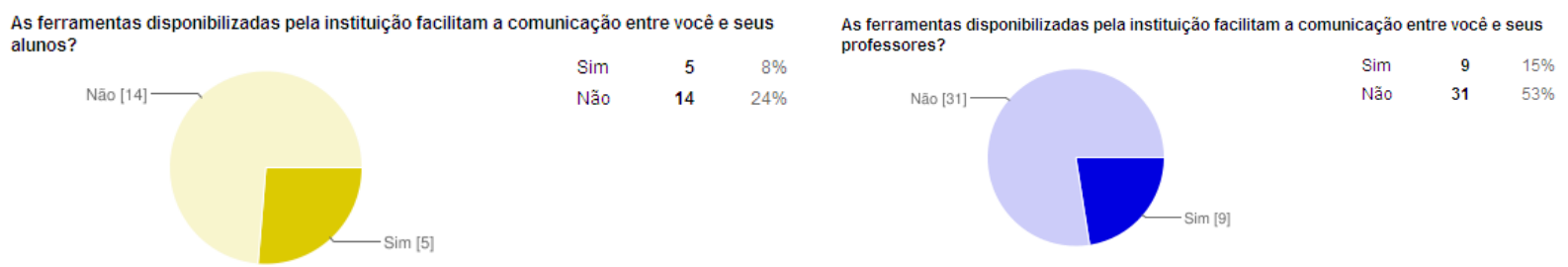

Figura 6 - Opinião de professores e acadêmicos sobre ferramentas de comunicação entre este e seus alunos.

\section{GERAÇÃO DE ALTERNATIVAS E DESENVOLVIMENTO}

A fase de geração de alternativas para uma interface web possui duas subfases. A fase anterior é a da arquitetura da informação da rede, onde serão definidas todas as páginas que o website conterá, e todos os caminhos possíveis que o usuário poderá percorrer. A próxima fase é a do desenvolvimento da interface onde se desenvolve um wireframe (uma espécie de layout sem cores, formada apenas por linhas e caixas) e depois se desenvolve o layout baseado no wireframe, adicionando cores e formas. A estrutura do site foi desenvolvida para que o usuário sempre percorra o menor caminho para obter o que ele deseja. 

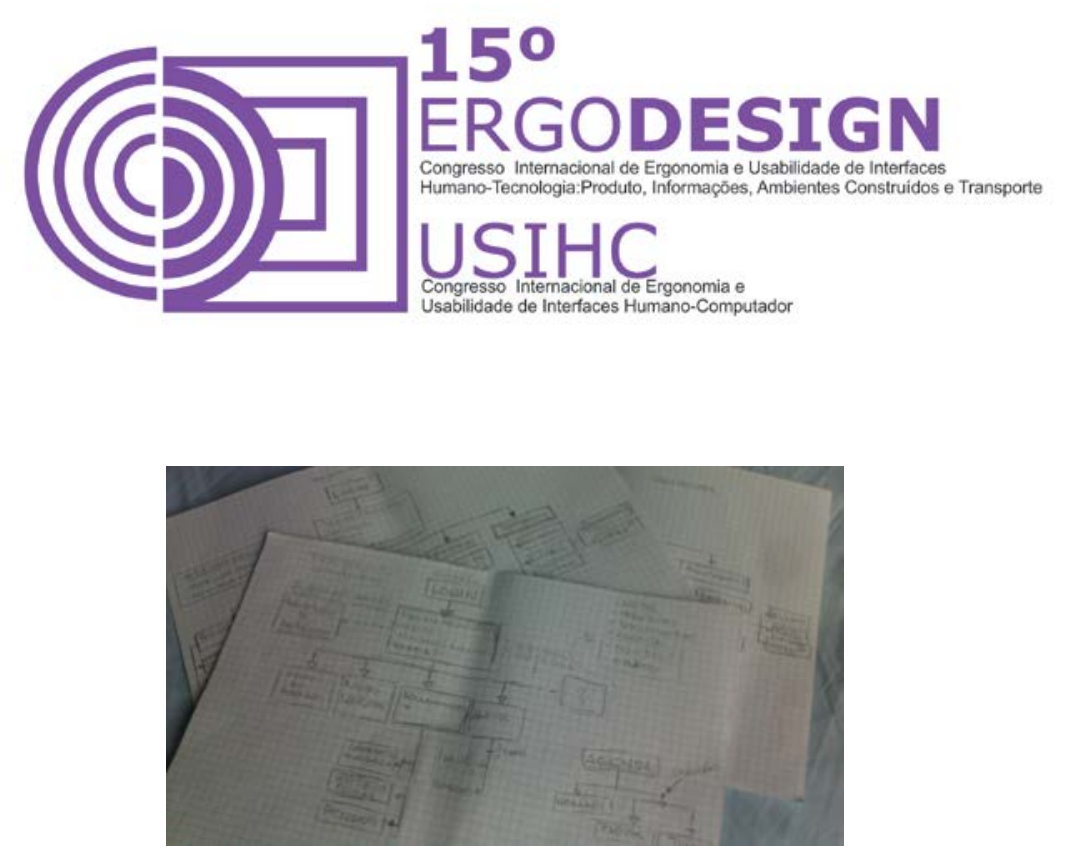

Figura 7 - Esboços da arquitetura da Informação.

Para chegar ao resultado esperado, dividiu-se o site em 3 áreas, informações iniciais, navegação e informação interiores. Essa divisão permitiu a organização do conteúdo de todo o website, facilitando a definição da arquitetura.

No organograma abaixo é possível notar as três divisões citadas anteriormente. Onde na área verde estão as Informações Iniciais, ou seja, as informações que o usuário receberá primeiro, na área azul está a área de Navegação, ou seja, os links para as demais páginas do website, e na área vermelho-escuro estão as Informações Interiores, ou seja, as informações que o usuário receberá após passar pela área-azul.
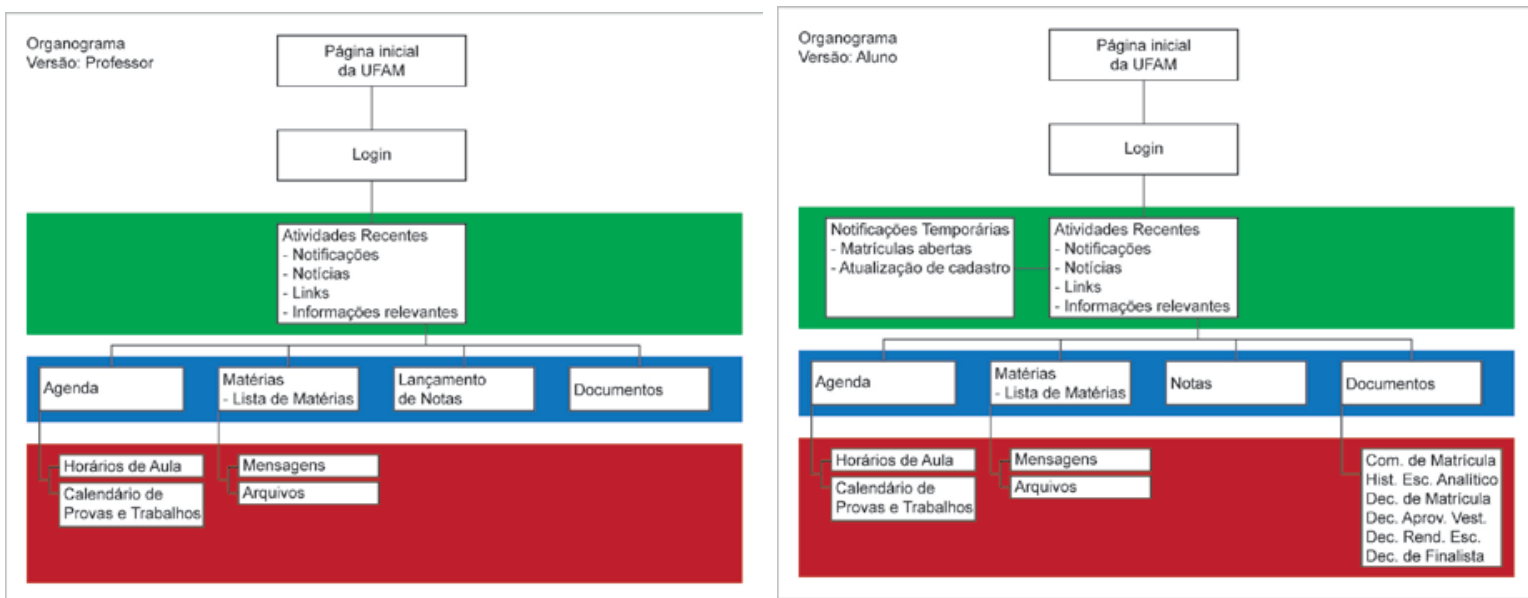

Figura 8 - Organograma da versão do professor e do aluno.

Iniciou-se o desenvolvimento do projeto, criando dois wireframes para a página de início do site, dentro os quais um foi escolhido pois procurou-se focar a atenção do usuário na coluna do meio, onde ele obterá as principais informações iniciais e interiores, sem deixar de oferecer atenção para as colunas laterais, a da esquerda com a área de navegação, e a da direita com um bloco de notícias da universidade e este ofereceu mais opções para o desenvolvimento do 


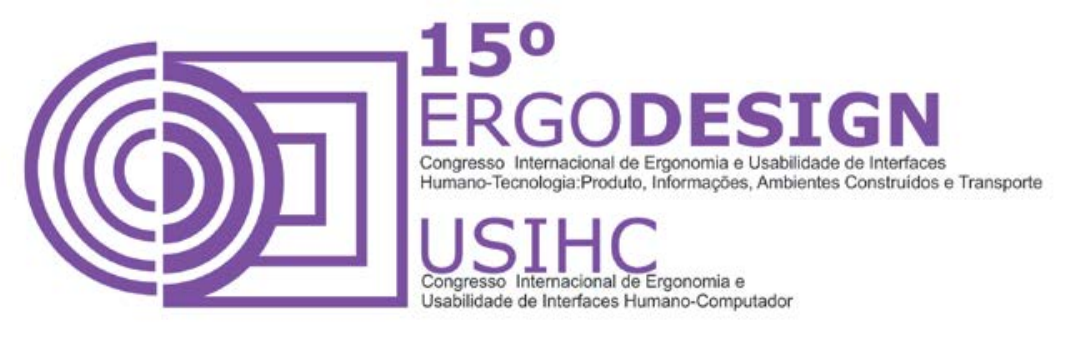

website além da maior similaridade com a interface de uma rede social. A partir disso, foram decididas quais cores iriam compor o website. As cores escolhidas foram Azul, Laranja, Preto e Verde nos seguintes tons:

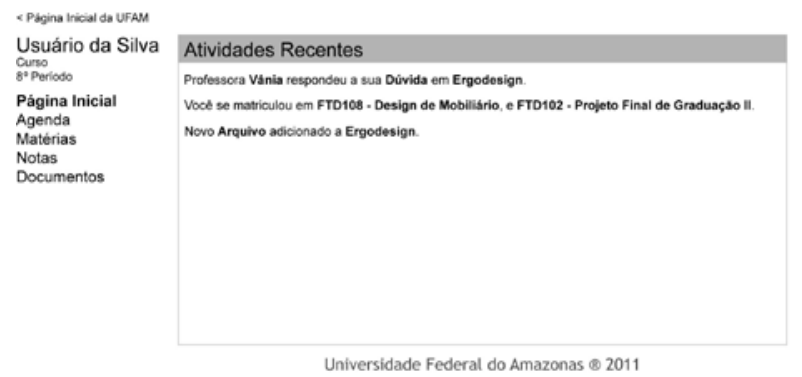

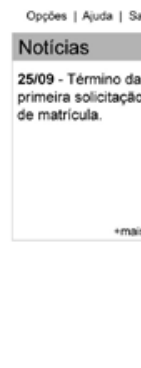

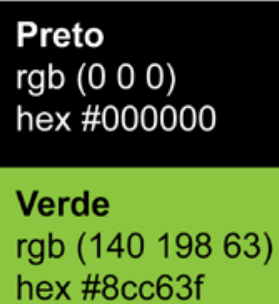

Figura 10 - Wireframe da segunda interface e Cores Escolhidas

Escolheu-se o Azul porque é a cor que representa a água, uma das principais riquezas da região amazônica, local da Universidade Federal do Amazonas. A cor Laranja representa a madeira, o que sustenta e alimenta o verde das árvores presentes em abundância na UFAM. O Preto foi escolhido por ser a fonte mais aconselhada para contrastar com fundos claros. $O$ Verde representa a floresta. A UFAM está dentro de uma das maiores reservas florestais urbanas do Brasil, tornando o verde uma das cores mais lembradas para representar a UFAM. Decidiu-se usar tons claros e cintilantes para que o usuário fique atento à página enquanto ele estiver usando-a.

O website foi pensado em cima de um grid de 960px (px, ou pixels, medida utilizada durante o desenvolvimento de websites, num monitor é o menor ponto que se é possível atribuir uma cor), por isso foi a largura máxima adotada. Todas as outras áreas foram também baseadas neste grid, sendo dividido em colunas de 60px e 20px.

Como família tipográfica escolheu-se as sem-serifa, e como fonte padrão Arial. Isto é, caso o computador de determinado usuário não possuir a fonte Arial, o navegador usará a fonte semserifa padrão do sistema operacional. Para o nome do usuário e curso definiu-se tamanho de fonte 20pt e para o menu de navegação 14pt. Para o texto das colunas central e direita definiuse como tamanho padrão para texto $15 \mathrm{pt}$ e para o título da coluna $20 \mathrm{pt}$. E para o menu de navegação da barra superior 13pt. 

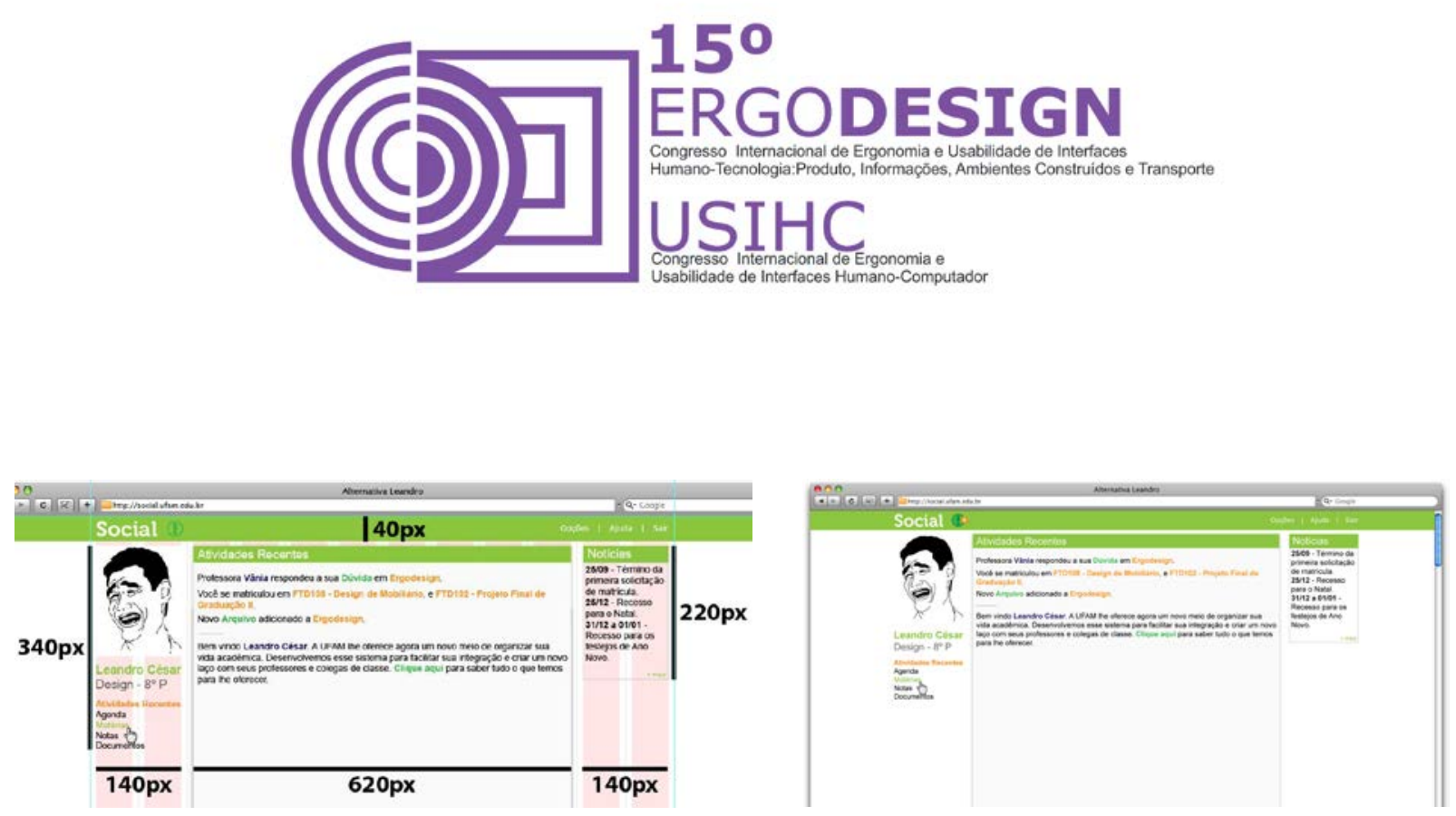

Figura 11 - Layout final.

\section{CONSIDERAÇÕES FINAIS}

O presente projeto, que contemplou o desenvolvimento de uma interface permitindo um sistema de comunicação entre aluno e professor, atendeu os seus objetivos propostos. Foi visto que a Universidade Federal do Amazonas está presente na internet, porém ela não utiliza dos recursos oferecidos que podem facilitar o trabalho entre acadêmico e professor. Com essa percepção foi possível definir que caminho seguir para a solução desse principal problema. A interface desen-volvida contemplou todos os objetivos propostos visando proporcionar ao usuário um sentimento de familiaridade e a continuidade deste projeto pode resultar em futuras mudanças na UFAM, não apenas na questão digital, mas também na questão social, como por exemplo, um sistema social que integre toda a universidade.

\section{REFERÊNCIAS BIBLIOGRÁFICAS}

ADOLPHO, Conrado. Redes sociais: tendência ou modismo. iMasters. 1 setembro 2009. Disponível em: <http://imasters.com.br/artigo/14115/midiasocial/redes_sociais_tendencia_ou_modismo/> Acesso em: 22 março 2011.

ANTONIOLI, Leonardo. Estatísticas, dados e projeções atuais sobre a internet no Brasil. To Be Guarany. 26 maio 2011. Disponível em: <http://www.tobeguarany.com/internet_no_brasil.php> Acesso em: 21 março 2011.

Backbone. Wikipédia, a enciclopédia livre. Disponível em: < http://pt.wikipedia.org/wiki/Backbone> Acesso em: 21 março 2011.

BARROS, Guilherme. Brasileiros passam 41 horas por semana em redes sociais. IG. 4 novembro 2009. Disponível em: <http://colunistas.ig.com.br/guilhermebarros/2009/11/04/brasileiros-passam-41-horas-porsemana-em-redes-sociais/>. Acesso em: 21 março 2011.

BOGO, Kellen Cristina. A história da internet - como tudo começou. 01 julho 2000. K-plus. Disponível em: <http://kplus.cosmo.com.br/materia.asp?co=11\&rv=Vivencia> Acesso em: 09 junho 2011.

Breve introdução à internet. Departamento de Física da Universidade de Coimbra. Disponível em: <http://nautilus.fis.uc.pt/softc/Read_c/destaque/inter.htm> Acesso em: 09 junho 2011. 


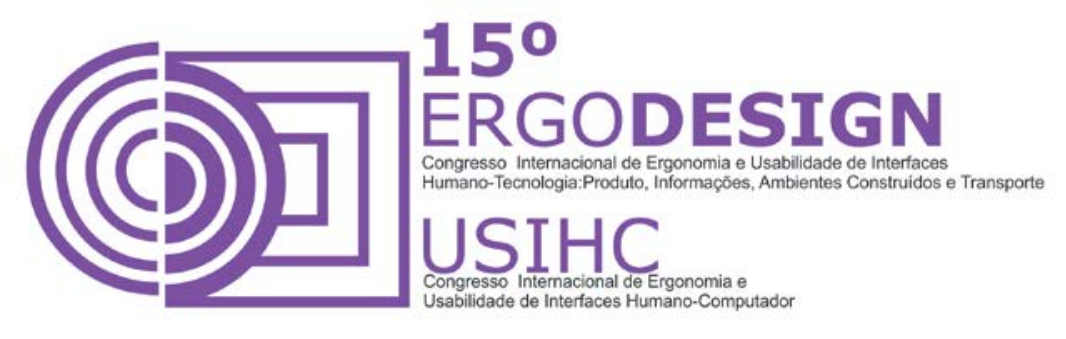

DEBIASI, Adam Esteves. Saiba qual foi a primeira rede social da internet. Social Media Empresarial. 8 abril 2010. Disponível em: <http://socialmediaempresarial.wordpress.com/2010/04/08/saiba-qual-foi-aprimeira-rede-social-da-internet/> Acesso em: 22 março 2011

Egito desliga a internet. O que acontece agora? Tento Aprender. 1 fevereiro 2011. Disponível em: <http://tentoaprender.blogspot.com/2011/02/egito-desliga-interneto-que-acontece.html> Acesso em: 22 junho 2011

Governo sírio desliga internet no país. Blogs POP Tech. 6 junho 2011. Disponível em: <http://blogs.pop.com.br/tecnologia/governo-sirio-desliga-internet-no-pais/> Acesso em: 22 junho 2011

Hipertexto. Wikipédia, a enciclopédia livre. Disponível em: < http://pt.wikipedia.org/wiki/Hipertexto> Acesso em: 21 março 2011.

How the world spends its time online. Visual Economics. 16 junho 2010. Disponível em: <http://www.visualeconomics.com/how-the-world-spends-its-time-online_2010-06-16>. Acesso em: 21 março 2011.

Internet. Wikipédia, a enciclopédia livre. Disponível em: <http://pt.wikipedia.org/wiki/lnternet> Acesso em: 21 março 2011.

Internet: serviços comuns. Universidade Federal do Pará. 9 fevereiro 2011. Disponível em: <http://www.ufpa.br/dicas/net1/int-ser.htm> Acesso em: 11 abril 2011.

Moderno dicionário da língua portuguesa online. UOL. Disponível em: <http://michaelis.uol.com.br> Acesso em: 21 março 2011.

MORAES, Ana Maria de; Mont'alvão, Claudia. Ergonomia: Conceitos e Aplicações. 4 ed. Rio de Janeiro. Ed. 2ab, 2010.

MORAES, Ana Maria de; ROSA, José Guilherme Santa. Avaliação e Projeto no Design de Interfaces. Teresópolis, RJ. Ed. 2ab, 2008.

O que é: backbone. G1. São Paulo. 03 julho 2008. Disponível em: < http://g1.globo.com/Noticias/0,,MUL634945-15524,00-O+QUE+E+BACKBONE.html> Acesso em: 21 março 2011.

PIMENTEL, Djenane. Orkut. Acessa.com. 21 setembro 2004. Disponível em: <http://www.acessa.com/informatica/arquivo/tecnologias/2004/09/21-orkut/> Acesso em: 22 março 2011.

Rede social. . Wikipédia, a enciclopédia livre. Disponível em: <http://pt.wikipedia.org/wiki/Rede_social> Acesso em: 22 março 2011.

Redes sociais equivalem a 15\% do tempo de navegação em junho. IDG NOW. 30 julho 2007. Disponível em: <http://idgnow.uol.com.br/internet/2007/07/30/idgnoticia.2007-07-30.2011442140/> Acesso em: 09 junho 2011.

RUDIO, Franz Victor. Introdução ao Projeto de Pesquisa Científica. 36 ed. Petropólis, RJ. Ed. Bozes, 2009.

ZEVALLOS Jr, Ruben. A história da internet. Artigonal. 24 janeiro 2009. Disponível em: <http://www.artigonal.com/ti-artigos/a-historia-da-internet-737117.html> Acesso em: 09 junho 2011. 